\title{
Update on birth route in case of cesarean section in a previous pregnancy
}

\section{AtualizaçÃo EM VIA DE PARTO EM CASO DE CESARIANA EM GESTAÇÃo ANTERIOR}

Sergio F. de Toledo ${ }^{1}$, Ricardo Simões ${ }^{1,2}$, Romulo P. Soares ${ }^{2}$, luca S. Bernardo ${ }^{2}$, Wanderley M. Bernardo ${ }^{2}$, Antônio J. Salomão ${ }^{1}$, Edmund C. Baracat ${ }^{1}$

1. What is the level of evidence of studies on birth route in case of cesarean section in a previous pregnancy?

a. Observational longitudinal studies and lack of controlled studies.

b. Several randomized controlled trials support the evidence.

c. Case reports only.

d. Expert opinion and narrative reviews.

2. The longitudinal observational studies comparing birth route in patients with cesarean section in a previous pregnancy are characterized by being:

a. Heterogeneous in terms of methodology to measure outcomes and presenting distinct follow-up durations.

b. Heterogeneous in terms of follow-up duration and homogeneous in outcome assessment.

c. Homogeneous in terms of methodology to measure outcomes and presenting distinct follow-up durations.

d. Homogeneous in terms of follow-up duration and heterogeneous in outcome assessment.

3. What are the characteristics associated with a favorable progress of trial of labor in patients with previous cesarean section?

a. Indication of the first cesarean section (for non-recurring factor) and vaginal delivery history, in the case of multiparous women with one previous cesarean section.

b. There are no characteristics favoring a trial of labor in this situation.

c. History of two cesarean sections.

d. Age greater than 35 years is the only factor to favorable progress.

4. Trial of labor in patients with previous cesarean section is:

a. A reasonable choice for pregnant women with one previous cesarean section (performed by means of transverse uterine section). b. A reasonable option for pregnant women presenting two previous cesarean sections (longitudinal scar).

c. Always indicated.

d. Always contraindicated.

5. Which is the birth route indicated for patients with cesarean section in a previous pregnancy?

a. There are still doubts about which type of delivery is better.

b. Cesarean delivery is always better.

c. Vaginal delivery is always better.

d. It varies with age; for patients older than 35 years, vaginal delivery is indicated.

\section{ANSWERS TO CLINICAL SCENARIO - TREATMENT OF ISOLATED LESIONS OF THE POSTERIOR CRUCIATE LIGAMENT [PUBLISHED IN RAMB 2015; 61(2)]}

1. What is the recommendation for the conservative treatment of the posterior cruciate ligament (PCL) injury?

It is recommended in isolated grade I and II ligament injuries (Alternative $\mathbf{C}$ ).

2. When is surgical reconstruction of the PCL indicated?

It is recommended in ligament injuries caused by bone avulsions (Alternative $\mathbf{B}$ ).

3. When surgery is recommended, is single tunnel PCL reconstruction similar to double tunnel reconstruction?

The functional and subjective results are similar (Alternative A).

4. Is use of homologous grafts recommended for reconstruction of the PCL?

Yes, the results are similar to those of autologous grafts (Alternative $\mathbf{D}$ ). 
5. How should postoperative treatment of PCL reconstruction be conducted?

Key points include extension immobilizer (Alternative $\mathbf{C}$ ).

ANSWERS TO CLINICAL SCENARIO - TREATMENT OF ABDOMINAL TUMORS USING RADIOTHERAPY [Published in RAMB 2015; 61(2)]

1. Is the irradiation of the abdominal area using conformal radiotherapy superior compared to conventional radiotherapy in pancreatic tumors?

Radiation entry into different angles shows better results (Alternative B).

2. Is the irradiation of the abdominal area using conformal radiotherapy superior compared to conventional radiotherapy in sarcomas?

There is better dose distribution in both children and adults (Alternative $\mathbf{C}$ ).
3. Is there less toxicity in the use of conformal radiotherapy compared to conventional radiotherapy for abdominal tumors in pancreatic cancer? There is less toxicity with conformal radiotherapy (Alternative A).

4. Is there less toxicity in the use of conformal radiotherapy compared to conventional radiotherapy for abdominal tumors in sarcomas?

Toxicity in the conventional technique can lead to the need for hospital intervention (Alternative $\mathbf{D}$ ).

5. Is there a difference in the effectiveness of conformal and conventional radiotherapy?

In stomach tumors relapse-free survival is higher in the conformal technique (Alternative A). 